\title{
In vitro penetration activity of ionic liquid-Gnetum gnemon seed extract loaded solid lipid nanoparticles
}

\author{
Elsa Trinovita ${ }^{1}$, Maesya Rachmawati², Sutriyo Sutriyo ${ }^{3 *}$, Abdul Mun'im ${ }^{4}$ \\ ${ }^{1}$ Faculty of Medicine, Universitas Palangka Raya, Central Borneo, Indonesia. \\ ${ }^{2}$ Directorate of Registration of Traditional Medicine, Health Supplement and Cosmetic, National Agency of Drug and Food Control, Jakarta, Indonesia. \\ ${ }^{3}$ Department of Pharmaceutical Technology, Faculty of Pharmacy, Universitas Indonesia, Depok, Indonesia. \\ ${ }^{4}$ Department of Pharmacognosy-Phytochemistry, Faculty of Pharmacy, Universitas Indonesia, Depok, Indonesia.
}

\begin{tabular}{|c|c|}
\hline ARTICLE INFO & ABSTRACT \\
\hline Received on: 11/10/2018 & Gnetum gnemon L. (Melinjo) seeds have high trans-resveratrol, which was known to have poor skin absorption ability. \\
\hline Accepted on: 27/06/2019 & This study was performed to evaluate the skin absorption of ionic liquid-melinjo seed extract (IL-MSE) loaded solid \\
\hline Available online: 05/10/2019 & $\begin{array}{l}\text { lipid nanoparticles (SLN). The SLNs was formulated with glyceryl monostearate as the lipid ingredient, ceteareth-25, } \\
\text { ceteareth-6, stearyl alcohol, an aqueous phase, and } 10 \% \text { melinjo seed extract. The SLNs were prepared by modification }\end{array}$ \\
\hline $\begin{array}{l}\text { Key words: } \\
\text { Franz cell diffusion, } \\
\text { Gnetum gnemon, ionic } \\
\text { liquid-melinjo seed extract, } \\
\text { solid lipid nanoparticles, } \\
\text { trans-resveratrol. }\end{array}$ & $\begin{array}{l}\text { of high-pressure homogenization. The polydispersity index (PDI), zeta potential, entrapment efficiency (EE), particle } \\
\text { size (PS), and morphological scanning electron microscopy (SEM) were evaluated. The in vitro penetration of IL-MSE } \\
\text { SLN was carried out by using the Franz cell diffusion method. The SLNs formulations showed an average of particles } \\
\text { size about } 794 \mathrm{~nm} \text {, and with high lipid and surfactant, the content could contributing to high entrapment efficiency } \\
\text { to almost } 89 \% \text {. Even though the polydispersity index of SLN was } 1.229 \text {, and zeta potential measurement was } 62.56 \\
\mathrm{mV} \text {. Up to } 45 \% \text { of the trans-resveratrol penetrated through the skin after } 8 \text { hours of the test run. The IL-MSE loaded } \\
\text { to SLNs could improve the absorption of trans-resveratrol through the skin. }\end{array}$ \\
\hline
\end{tabular}

\section{INTRODUCTION}

Melinjo (Gnetum gnemon L.) is a plant which belongs to the family of Gnetaceae and is grown in many regions in Malaysia, Indonesia, and the Philippines. In Indonesia, the seeds of melinjo are usually treated as the basic material in making a food called "emping" or "paddy oat." In addition, the peel of melinjo can be processed into vegetable and even most of as waste (Barua et al., 2015). Several studies have been conducted, and it is known that the seeds of melinjo contain various stilbenoid group compounds including trans-resveratrol, gnemonoside $\mathrm{A}$, gnemonoside $\mathrm{C}$, gnemonoside $\mathrm{D}$, gnetin $\mathrm{C}$ (dimer of resveratrol), and gnetin $\mathrm{L}$ (Kato et al., 2009). Tani proved that melinjo seeds contain higher transresveratrol than grape skin or seeds which are previously known

\section{"Corresponding Author}

Sutriyo Sutriyo, Department of Pharmaceutical Technology, Faculty of Pharmacy, Universitas Indonesia, Depok, Indonesia.

E-mail: sutriyo@farmasi.ui.ac.id to have a higher content of trans-resveratrol (Casazza et al., 2010; Tani et al., 2014).

Compared to other stilbenoid group compound, transresveratrol contained in the seeds of the melinjo has been widely shown some pharmacological activities, one of them as an antioxidant. Trans-resveratrol is also widely used in cosmetic preparation because of its efficacy as an anti-aging and skin lightening. It also works to inhibit the formation of melanin by inhibiting the tyrosinase (Lee et al., 2014). To provide this topical effect, an active ingredient relies heavily on its ability to penetrate through the skin. The skin penetration of an active ingredient is determined by its formulation design (Scognamiglio et al., 2013). The ability of resveratrol as the active ingredient is limited due to the low ability of water absorption; bioavailability is low due to the high chemical instability due to exposure to sunlight. It can convert the trans form into a cis form which is less active (Davidov et al., 2014).

Nowadays, there are some different delivery systems that have been investigated as potential approaches to improve bioavailability by increasing skin penetration of active ingredients, 
such as lipid nanoparticles, cyclodextrin-based nanosponges, polymeric lipid-core nanocapsules, and transfersomes (Ansari et al., 2011; Detoni et al., 2012; Gokce et al., 2012; Scognamiglio et al., 2013). Solid lipid nanoparticles (SLN) are a nanoparticle carrier that is widely studied and used in cosmetic and pharmaceutical fields. In recent years, SLN has been studied as a promising topical delivery system for various beneficial effects of trans-resveratrol, such as film formation and controlled occlusion, enhancement of chemical stability of actives, skin bioavailability, and also improvement of skin hydration and modulation of skin targeting (Gocke et al., 2012). Thus, it can also look up as a topical delivery system for ionic liquid-melinjo seed extract (IL-MSE) and give a beneficial effect of trans-resveratrol on the skin. This research was conducted to get an insight into the penetration ability of IL-MSE loaded to solid lipid nanoparticle system through the skin using the Franz cell diffusion method.

\section{MATERIALS AND METHOD}

\section{Plant materials and reagents}

The seeds of melinjo used in this study were collected and obtained from the area of Pandeglang-Serang, Banten, (Indonesia) and have been through the process of drying and shelling. Characteristic of the seeds of melinjo used as the research sample is fresh and red color. The trans-resveratrol standard was purchased from Sigma-Aldrich Pte. Ltd (Singapore). [Bmim]Br were obtained from Chengjie Chemical Co. Ltd (Shanghai-China). Glycerol monostearate, Ceteareth-25/Cremophor ${ }^{\circledR} \mathrm{A} 25$, and Ceteareth-6 dan Stearyl alcohol/Cremophor ${ }^{\circledR}$ A6 were generously supplied by BASF GmBH (Germany). All high-performance liquid chromatography (HPLC) reagents and the other chemical ingredient were purchased from Merck (Germany).

\section{Sample preparation}

The outer skin of melinjo was peeled and then washed with water until clean and then dried for 6 days with a covered black cloth with the help of sunlight. The use of black cloth to avoid direct contact with sun exposure. Then the seeds of melinjo were milled using a tool grinder to be crushed. The dried melinjo powder was first separated for fat using a solvent of n-hexane for 24 hours, then after it is filtered. The residues were extracted using $2.5 \mathrm{M}[\mathrm{Bmim}] \mathrm{Br}$ with appropriate microwave-assisted extraction (MAE) condition.

\section{Production of IL-MSE}

Defatted melinjo seed powder was extracted using an ionic liquid with a ratio of $1: 15 \mathrm{~g} / \mathrm{ml}$ [Bmim] Br at a concentration of 2.5 M. MAE (Modified Modena MV-3002) method was performed for 10 minutes with $10 \%$ microwave power. The obtained extract was filtered with a Buchner vacuum filter. The filtrate was collected, and ethyl acetate was added with the same volume ratio. The mixed solution was shaken using a magnetic stirrer for 5 minutes. The ethyl acetate phase was separated by separating funnel. The ethyl acetate phase was collected, evaporated using a rotary vacuum evaporator (Buchi, Switzerland), and dried using an oven vacuum (Jisico J-DVO 2) at $40^{\circ} \mathrm{C}$ for 24 hours.

\section{Preparation of IL-MSE loaded to SLN}

The SLN dispersion was prepared by the high-pressure homogenization (HPH) method with modification. The solid lipid was melted at $75^{\circ} \mathrm{C}$ temperature, and $10 \%$ of IL-MSE was added to the melted lipid. A hot surfactant solution was prepared by mixing Cremofor A25 (4\%) and Cremofor A6 (0.8\%) in a preheated aquadest at $75^{\circ} \mathrm{C}$. The surfactant solution was added to the melt in the lipid phase and stirred until homogeneous using a homogenizer (Edmund Buhler $\mathrm{GmBH}$ ) at 3,000 rpm for 15 minutes. The preemulsion dispersion was further homogenized under stirring at $30,000 \mathrm{rpm}$ for 5 minutes to form the nanoparticles.

\section{Measurement of particle size and zeta potential}

The polydispersity index (PDI), mean particle size (PS), and zeta potential of all formulations in this study were analyzed by photon correlation spectroscopy with a Delsa Nano (Beckman Coulter). PS and PDI of all the formulations were calculated using the equation of Stokes-Einstein on the scattering angle of $90^{\circ}$ under the temperature of $25^{\circ} \mathrm{C}$.

\section{Measurement of scanning electron microscopy}

The morphology of the IL-MSE loaded SLN was determined using scanning electron microscopy (SEM) (Model LEO 1455VP, LEO). Lyophilized SLN was applied evenly on a conductive tab on a stub and then sputter coated with gold in a cathodic evaporator.

\section{Measurement of entrapment efficiency}

The entrapment efficiency (EE) of SLN dispersion was measured using the centrifugal tubes. Lipid nanocarrier dispersions were added into tubes and centrifuged using centrifugation (Kubota 5100, Japan) at 3,000 rpm for 15 minutes. The concentrations of trans-resveratrol in the dispersion (total resveratrol) and the filtrate (free resveratrol) were measured using HPLC. Based on the calculation of EE below explains that the Wi is the total amount of trans-resveratrol, while the $\mathrm{Wf}$ is the amount of free trans-resveratrol:

\section{High-performance liquid chromatography (HPLC)}

HPLC (Shimadzu, Japan) method was used to analyze trans-resveratrol content. Each sample was injected as much

$$
E E(\%)=\frac{(\mathrm{Wi}-\mathrm{Wf})}{\mathrm{Wi}} \chi 100 \%
$$

as $20 \mu \mathrm{l}$ into a YMC HPLC column $(150 \mathrm{~mm} \times 4.6 \mathrm{~mm}, 5 \mu \mathrm{m})$ with a wavelength set at $306 \mathrm{~nm}$ and a flow rate of $1.0 \mathrm{~mL} / \mathrm{min}$ through the UV-20AT UV detector (Shimadzu, Japan) in isocratic conditions. Each sample has three repetitions. The mobile phase was a mixture of water: acetonitrile: acetic acid with a ratio of 75:25 and adjusted to $\mathrm{pH} 3$ by using means of $\mathrm{pH}$ meter (Eutech Instruments Pte Ltd, Singapore).

\section{Preparation of SLN loaded based gel}

Preparation of Gel-SLN was done by dispersing the carbomer into the distilled water and stirred at $500 \mathrm{rpm}$ until homogeneous. Propylene glycol was added to the dispersion and neutralized by sodium hydroxide under gentle stirring until $\mathrm{pH}$ reaches 5.40. SLN dispersion was mixed to the dispersion, and the aqueous solution containing other ingredient was added to gel base and homogenized in a high-speed stirrer using homogenizer (Edmund Buhler $\mathrm{GmBH}$ ) to form a homogeneous and thick gel mass. The conventional gel was prepared by simply dispersing IL- 
MSE into the gel matrix. Table 1 shows the formulation of the gel composition.

\section{Rheological measurement}

Brookfield Viscometer was used for the measurement of rheological. Before the measurement of dial readings using $\mathrm{T}-\mathrm{C}$ spindles at $0.5,1,2.5$, and $5 \mathrm{rpm}$ was carried out; the sample was placed in a Beaker glass and allowed to equilibrate for 5 minutes. Repeat this with the same steps. At each speed setting, the reading of the corresponding dial reading on the viscometer was documented.

\section{In vitro penetration test using modified Franz diffusion cell}

This research was approved by the Institutional Animal Care Committee, Faculty of Medicine, Universitas Indonesia (Ethic No. 728/UN2.F1/ETIK/2016). In vitro penetration test was conducted in a modified Franz diffusion cell over 8 hours period. The sink condition was maintained using phosphate buffer $\mathrm{pH} 7.4$ in the receptor compartment, and the temperature was maintained at $32 \pm 1^{\circ} \mathrm{C}$ with a circulating water bath. Each one $\mathrm{mL}$ of sample was withdrawn from the receptor compartment at appropriate time intervals. Each sample containing trans-resveratrol was determined using the HPLC method.

\section{RESULTS AND DISCUSSION}

\section{Preparation of IL-MSE loaded to SLN}

In this research, the particle size was reduced by homogenization method at $30,000 \mathrm{rpm}$ for 5 minutes. The appearance of SLN suspension was described to have a light brown color with a distinctive aromatic scent, as shown in Figure 1. SLN formulation of IL-MSE shows the value of the zeta potential 62.56 $\mathrm{mV}$, as shown in Figure 2. The measurement of the zeta potential of a colloid needs to be done because the value of the zeta potential gives information about the stability of a colloid during storage. It shows that the system SLN shows the value of zeta potential above $60 \mathrm{mV}$ and can be shifted to the range of stability that is excellent. This is by the zeta potential (absolute value) above 60 $\mathrm{mV}$ indicating that it has excellent stability, the zeta potential above $30 \mathrm{mV}$ suggests physical stability, under $20 \mathrm{mV}$ showing the stability of the limited, and lower than $5 \mathrm{mV}$ associated with evidence of significant agglomerations (Muller, 1996).

Table 1. Formulation of SLN Based Gel and Blank Gel

\begin{tabular}{llcc}
\hline Composition & Function & $\begin{array}{c}\text { Formula I } \\
(\% \text { w/w })\end{array}$ & $\begin{array}{c}\text { Formula II } \\
(\% \mathbf{w} / \mathbf{w})\end{array}$ \\
\hline IL-MSE & Active ingredient & - & 1 \\
IL-MSE Loaded SLN & Active ingredient & 10 & - \\
Carbomer & Gelling agent & 0.4 & 0.4 \\
Propylene Glycol & Humectant & 10 & 10 \\
Sodium Metabisulfite & Antioxidant & 0.1 & 0.1 \\
Pottasium Sorbate & Preservative & 0.15 & 0.15 \\
Disodium EDTA & Chelating & 0.01 & 0.01 \\
Sodium Hydroxide & pH adjuster & 0.5 & 0.5 \\
Parfume & Flavor & 0.1 & 0.1 \\
Aqua & Solvent & Ad 100 & Ad 100 \\
\hline
\end{tabular}

The research also performed the SEM analysis to determine the morphology of the established SLN system. Figure 3 shows the results of SEM analysis of the IL-MSE loaded to SLN system with $100 \times$ and $400 \times$ magnification. The results of the SEM analysis showed the particles of SLN resveratrolshaped spherical with a particle size of $>200 \mathrm{~nm}$. IL-MAE is one of the methods of extraction used in the process of separation of a bioactive compound from the plants that show increased levels of bioactive compounds as compared with conventional extraction methods (Trinovita et al., 2017).

One of them is by using the method of IL-MAE as a method of extraction of trans-resveratrol from the seeds of melinjo. Based on the results of previous research with optimum conditions parameters include ionic liquid $[\mathrm{Bmim}] \mathrm{Br}$ concentration of $2.5 \mathrm{~mol} / \mathrm{l}$; sample/liquid ratio of $15 \mathrm{~g} / \mathrm{ml}$; extraction time of 10 minutes and microwave power of $10 \%$ with the extraction yield of trans-resveratrol was $1.34 \mathrm{mg} / \mathrm{g}$ (Ayuningtyas et al., 2017). Melinjo contains an abundant stilbenoid compound such as gnetin $\mathrm{L}$, gnemonosides $\mathrm{D}$, gnemonosides $\mathrm{C}$, gnemonosides $\mathrm{A}$, gnetin $\mathrm{C}$, and also trans-resveratrol. The study also conducted to get information about the biological activity of each compound and it was concluded that melinjo extract with those constituents is used as a supplementary to health (Kato et al., 2012).

\section{Preparation of SLN-based gel}

SLN-based gel used carbomer as a gelling agent. Carbomer was selected as the gelling agent because it has better properties in releasing the active substance compared to another gelling agent. The use of carbomer was also expected to produce clear, water-soluble, and low toxicity. Thus, it will not irritate the skin. Besides the facts that carbomer has a good spread on the skin, cooling effect, it does not clog the pores, easy to wash with water, and is good in releasing the active substance. Carbomer concentration used was $0.4 \%$ to form a gel with a moderate

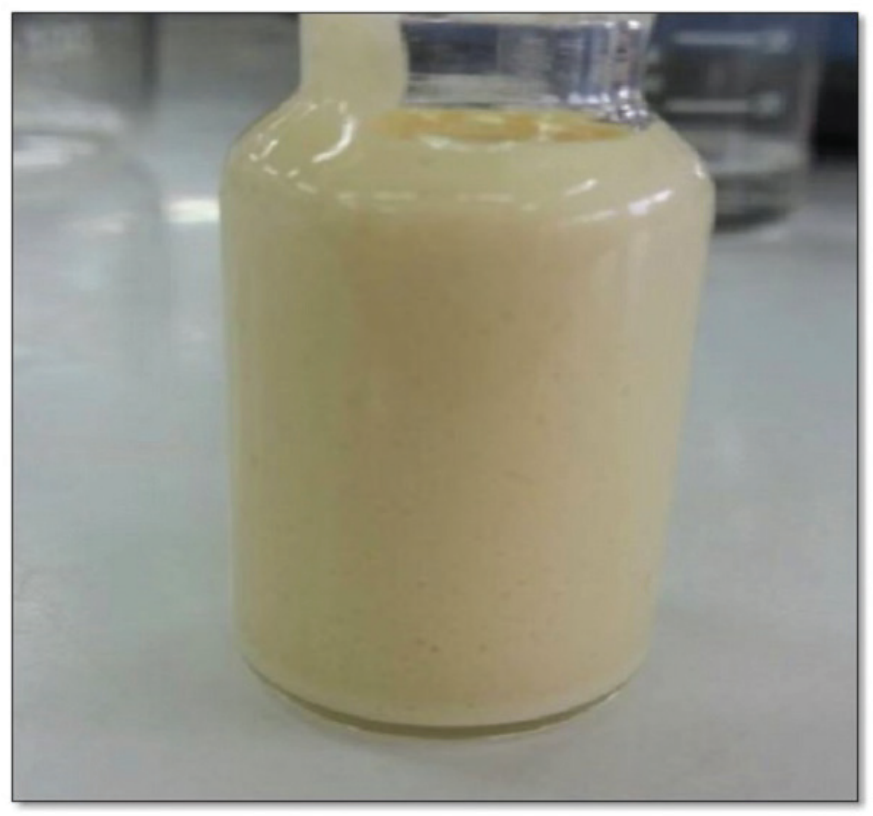

Figure 1. IL-MSE Loaded SLN System 


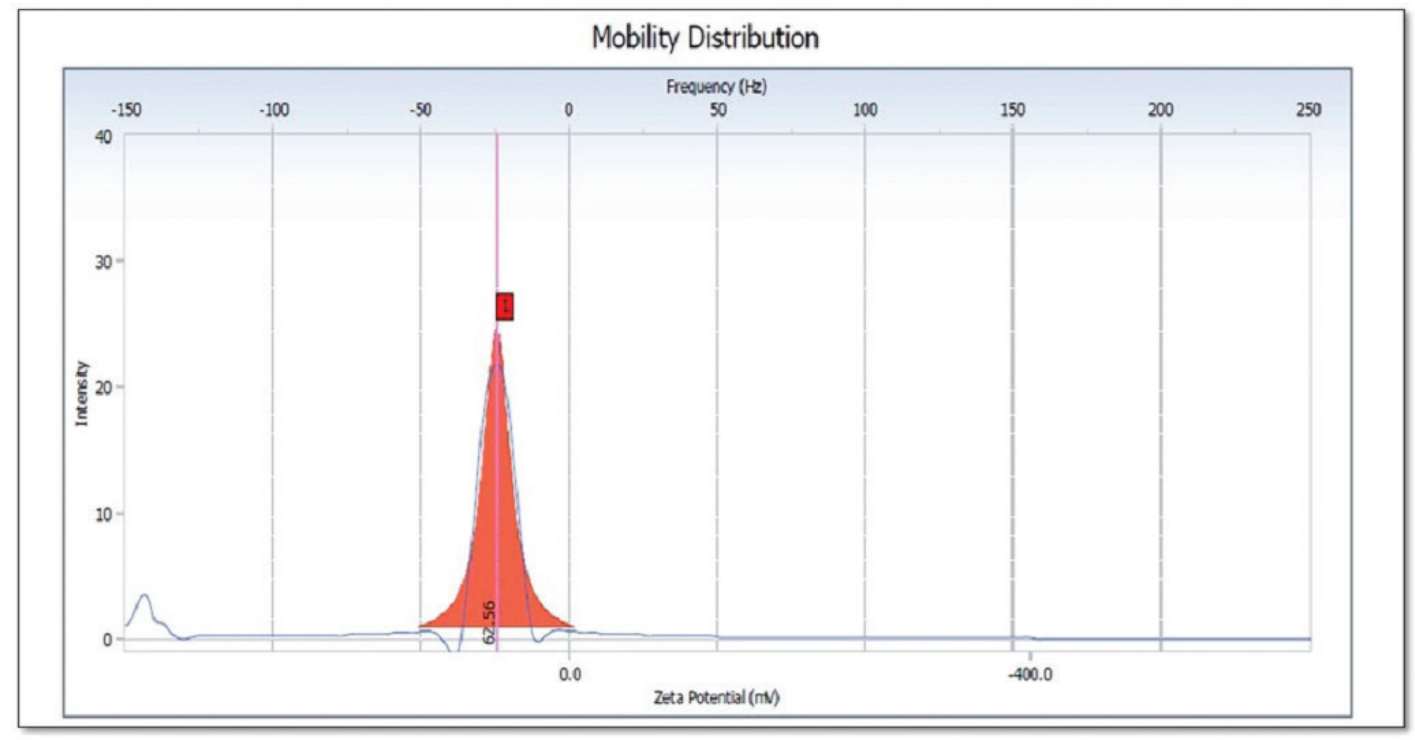

Figure 2. Zeta Potential of the Ultrafiltrated Dispersions of SLNs

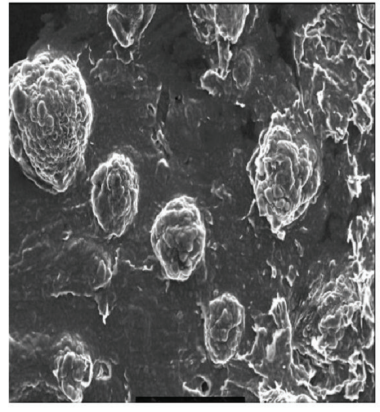

A

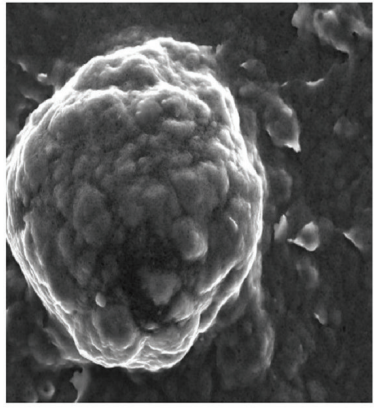

Figure 3. SEM of IL-MSE Loaded SLN with (A) 100x and (B) 400x magnification

viscosity to facilitate a good spreading during application, as well as providing a good physical appearance and preparation $\mathrm{pH}$ corresponds to skin physiological $\mathrm{pH}$. The other aqueous additives were added to a homogeneous gel basis, and the appearance of SLN-based gel and the blank gel was shown in Figure 4.

Trans-resveratrol, a component that has been widely studied is easily degraded or oxidized by alteration of $\mathrm{pH}$, extreme temperatures, and exposure to sunlight (Davidov et al., 2014). Thus, in the preparation of SLN, a method that does not affect the stability of the active substance was selected, which is HPH. This method is quite reliable and easier both for small-scale and to be increased on a larger scale due to the market availability of homogenizer in various sizes (Saratchandiran et al., 2012). IL-MSE containing trans-resveratrol will not degrade on the manufacturing process when the temperature up to $75^{\circ} \mathrm{C}$, this may be due to its thermostable characteristic if it was heated up to $100^{\circ} \mathrm{C}$ (Liazid et al., 2007).

The lipid selection for making the SLN matrix should be carefully selected because it could affect the particle size, entrapment efficiency, storage stability, and drug release. Lipid selection should also consider the ability in dissolving active component. glyceryl monostearate (GMS) was chosen as a solid

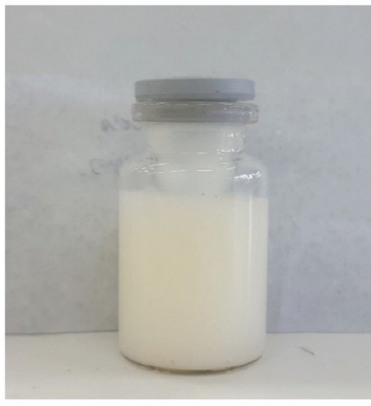

A

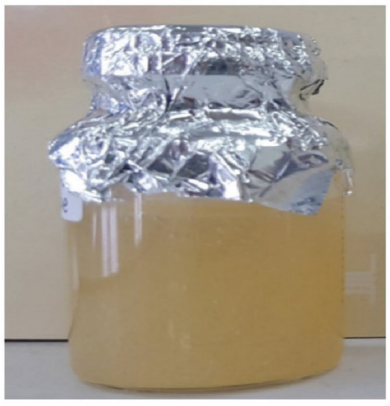

B
Figure 4. The appearance of SLN Based Gel (A) and Blank Gel (B)

matrix because it was widely used as a lipid material for the incorporation of active component into lipid nanocarrier, and it has a high solubility to trans-resveratrol. SLN preparations using the HPH method require the use of surfactants to stabilize the preparation. Surfactants used in this study were A25 and A6 as co-surfactants. Mixtures of surfactants and cosurfactants are more effective in stabilizing compared to the use of single surfactant, and it is also capable of reducing the particle size system. The use of a combination of A25 and A6 surfactants optimally stabilizes the SLN system because their use was proven to prevent agglomeration of SLN systems for four weeks (Sun et al., 2014).

\section{Rheological measurement}

In this research, the rheological measurement of SLNbased gel and the blank gel was performed using a stress control rheometer (Viscometer Brookfield). Furthermore, the continuous shear investigations have been applied to characterize the developed semi-solid formulations, evaluating the shear stress as a function of shear rate. This study begins with rheological measurements by applying the shear rate from 0 to 0.05 seconds $^{-1}$ and back to 0 seconds ${ }^{-1}$, and the yield shear stress and the viscosity measured. This was done to determine if the system is thixotropic or not. 
The obtained average value from each of the readings is used to calculate the viscosity. Based on the rheological measurement, Figures 5 and 6 show that SLN-based gel and blank gel have rheological properties of pseudoplastic thixotropic.

Particle size is the main thing to be evaluated since the term nanoparticles are indicated by the particle size of a solid or semisolid colloid within the range of 50-1,000 nm (Muller et al., 2002). Evaluation of the value of the size of the particles is used to determine the effect of the ratio of lipid-drug on the physical properties of SLN. SLN with the composition of $10 \%$ GMS, $4 \%$ $\mathrm{A} 25$, and $0.8 \%$ A6 gives particle measurement result with an average particle size which is $794.6 \mathrm{~nm}$, while the PDI is equal to 1.229 . According to the previous research showed that smaller particle size $(<200 \mathrm{~nm})$ could increase the penetration in the tumor tissue which can lead to the desired Enhanced Permeation and Retention effect (Teja et al., 2018). The measurement of particle size by preparing nanoparticles as a form of drug delivery to the target organ addressed in the body through a network of capillary vessels. In this experiment shows the result of the size of the larger particles $(>200 \mathrm{~nm})$ and affect the absorption of the nanoparticles and the movement of intracellular. This leads to a decrease in the penetration of trans-resveratrol that can reduce the desired effects. Low levels of trans-resveratrol that penetrated strongly by the composition of lipid contained in the system of SLN so that the preparation of the gel of SLN produced in accordance to the purpose of topical use and showed activity in the epidermis

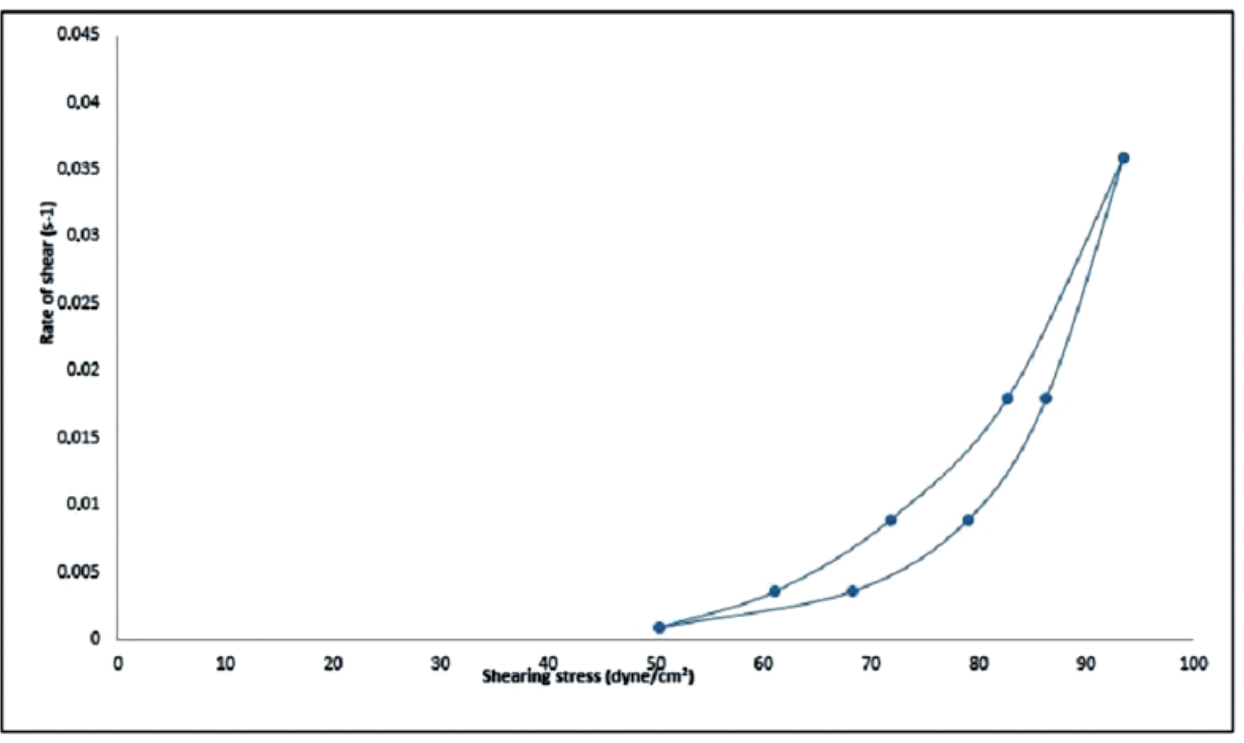

Figure 5. Rheogram of IL-MSE SLN Based Gel

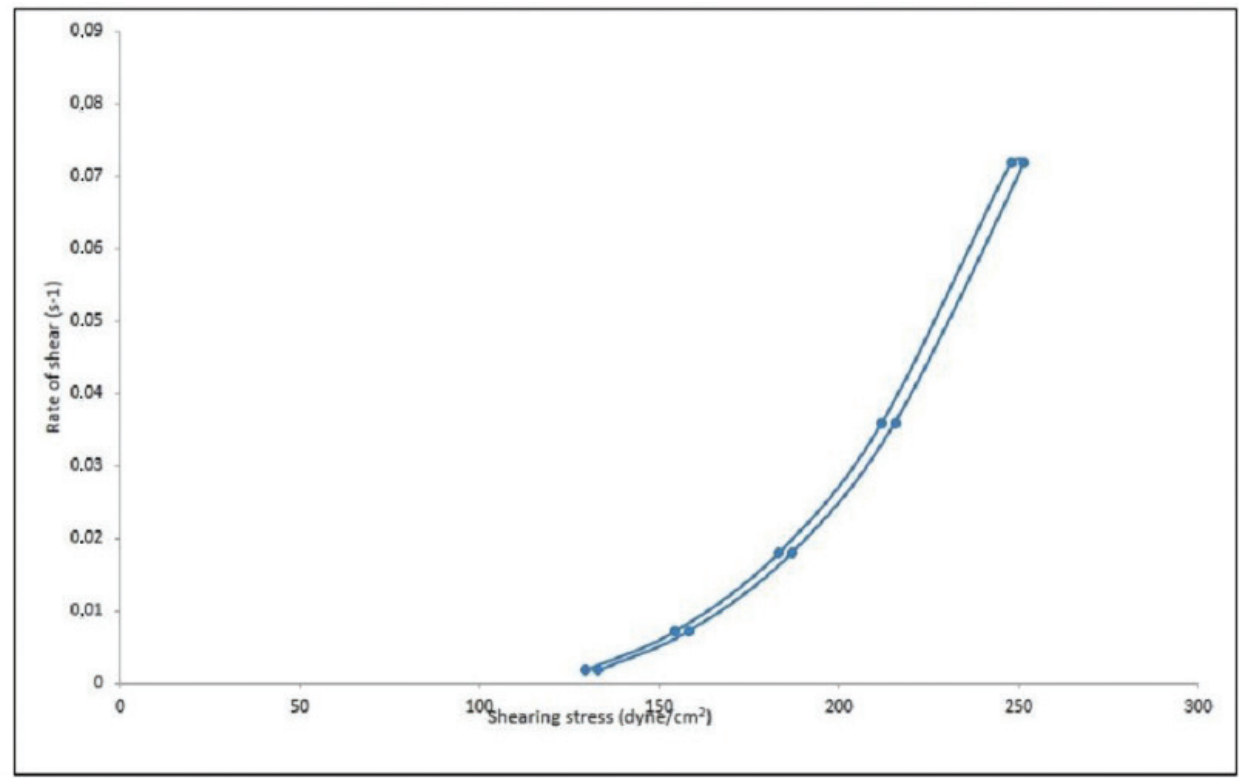

Figure 6. Rheogram of Blank Gel 
layer of the skin. This is by the previous discussion, which showed that the concentration of chitosan increased could enlarge the size of the particles due to the accumulation of large molecules in nanocarrier (Teja et al., 2018).

Increasing the amount of fat in the formula SLN 5-10\% in many cases produce a particle size of SLN with a large particle size distribution. The greater the number of GMS in the formula of the SLN, then the diameter of the particles and the PDI tend to be more enlarged. This is because the increase in the fat composition and the number of surfactants that remain will form a larger particle size because the surface area that can be covered by a surfactant is not increased. Meanwhile, the number of fat increases resulting in the presence of the surface fat that can not be covered by the surfactant so that the fat will agglomerate and generate a larger particle size (Mehnert et al., 2001).

The particle size distribution of a polymer can be expressed based on the value of PdI. The PdI is higher with a previous theory, which explains that the PdI greater than 0.7 is considered to be too polydisperse to measure the size distribution (Stetefeld et al., 2016). The greater the concentration of SLNbased gel then the size of the particles obtained by the smaller. This is due to the diameter of the particles is influenced by the concentration of SLN-based gel that is used.

\section{In vitro penetration test using Franz cell diffusion}

In evaluating the skin targeting potential of all developed formulations, to provide useful data for forecasting resveratrol bioavailability in the skin, the permeation ability of resveratrol from nanocarriers into and through the skin could be studied both using in vitro or in vivo method. This research was to evaluate the penetration ability of preparation through the skin using abdomen rat skin with the Franz cell diffusion method. Figure 7 shows the total cumulative amount of trans-resveratrol penetrated across rat skin as a function of time from two different formulations.
The in vitro skin penetration of the drug is mainly assessed by its flux $\left(\mu \mathrm{g} / \mathrm{cm}^{2} /\right.$ hour). This was calculated from the slope of the linear steady portion of the total cumulative amount released versus time (minute) plot, as shown in Figure 7. In the present work, the transdermal flux of SLN-based gel had an average flux value of $1.45 \pm 0.04 \mu \mathrm{g} / \mathrm{cm}^{2} /$ hour, while IL-MSE blank gel had an average flux value of $0.36 \pm 0.2 \mu \mathrm{g} / \mathrm{cm}^{2} /$ hour. It showed that the SLN system was able to fix the permeability of trans-resveratrol through the skin. This condition causes the number of trans-resveratrol penetrated in SLN gel preparation after 8 hours to be higher than blank gel, which is $45.80 \%$, while for blank gel, it only reaches $14.06 \%$.

Zeta potential measurement was conducted to analyze the stability of colloidal dispersion during its storage period and is also a good predictor of the gelation phenomenon (Mehnert et al., 2001). The zeta potential reflects the potential charge of the particle and it is affected by the particle composition as well as the dispersing medium. Zeta potential shows the charge of colloidal particles associated with electrostatic resistances between particles that could prevent the occurrence of aggregation of colloidal particles. A colloidal dispersion can be stated as stable at storage if its potential zeta value is greater than $+30 \mathrm{mV}$ or less than $-30 \mathrm{mV}$ (Rigon et al., 2016).

Entrapment efficiency is an index to determine the capacity of nanocarriers for topical application. The entrapment efficiency SLN was done by separating procedure of IL-MSE from the system. Separation method was performed using the high-speed centrifugation, which is the active substance that is not entangled in the SLN will freely pass through the membrane, while the SLN particles trapped in the lipid layer formed. The content of transresveratrol in the supernatant was then identified using a validated HPLC method. Figure 8A shows the chromatogram of standard trans-resveratrol, while Figure $8 \mathrm{~B}$ shows the chromatogram of trans-resveratrol in the SLN. The EE value of the SLN system was determined as $89.46 \%$ due to the poor solubility of trans-

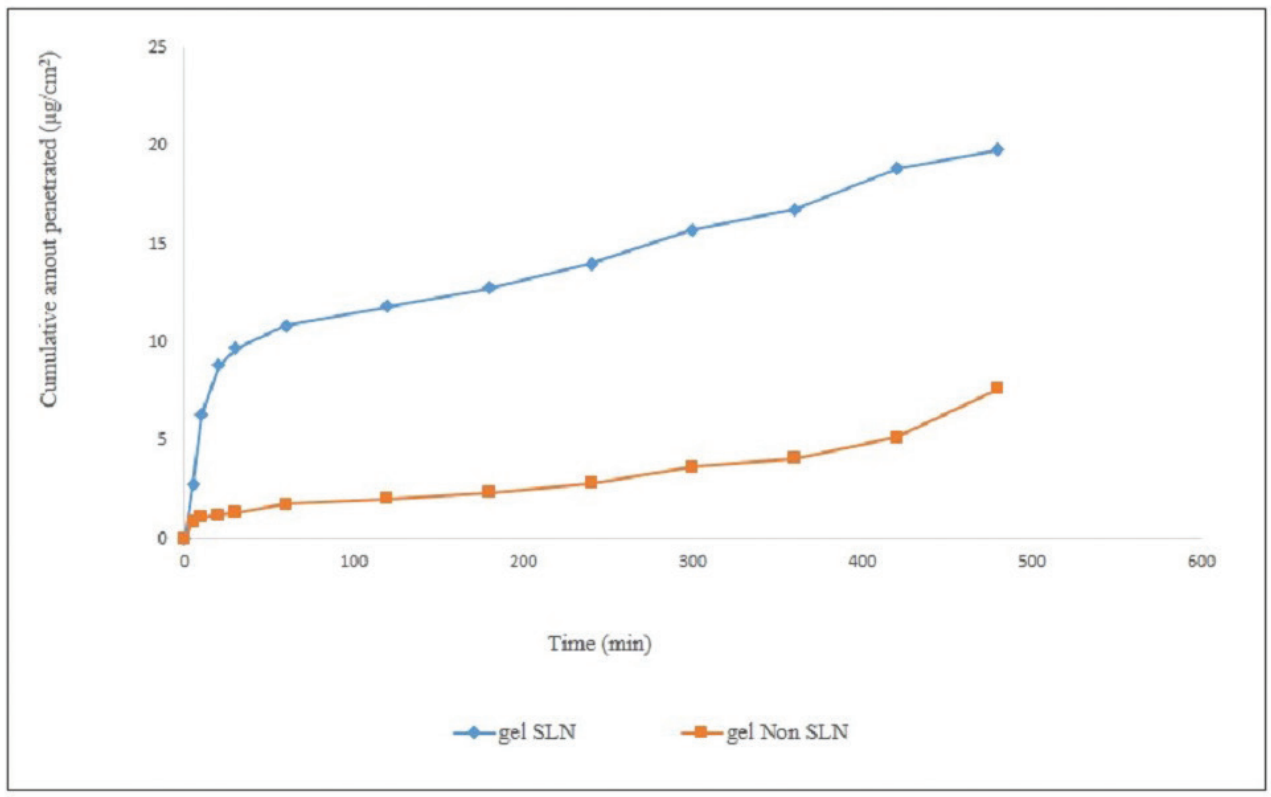

Figure 7. Cumulative Amount of Trans-Resveratrol Across Rat Skin of Two Different Formulation 

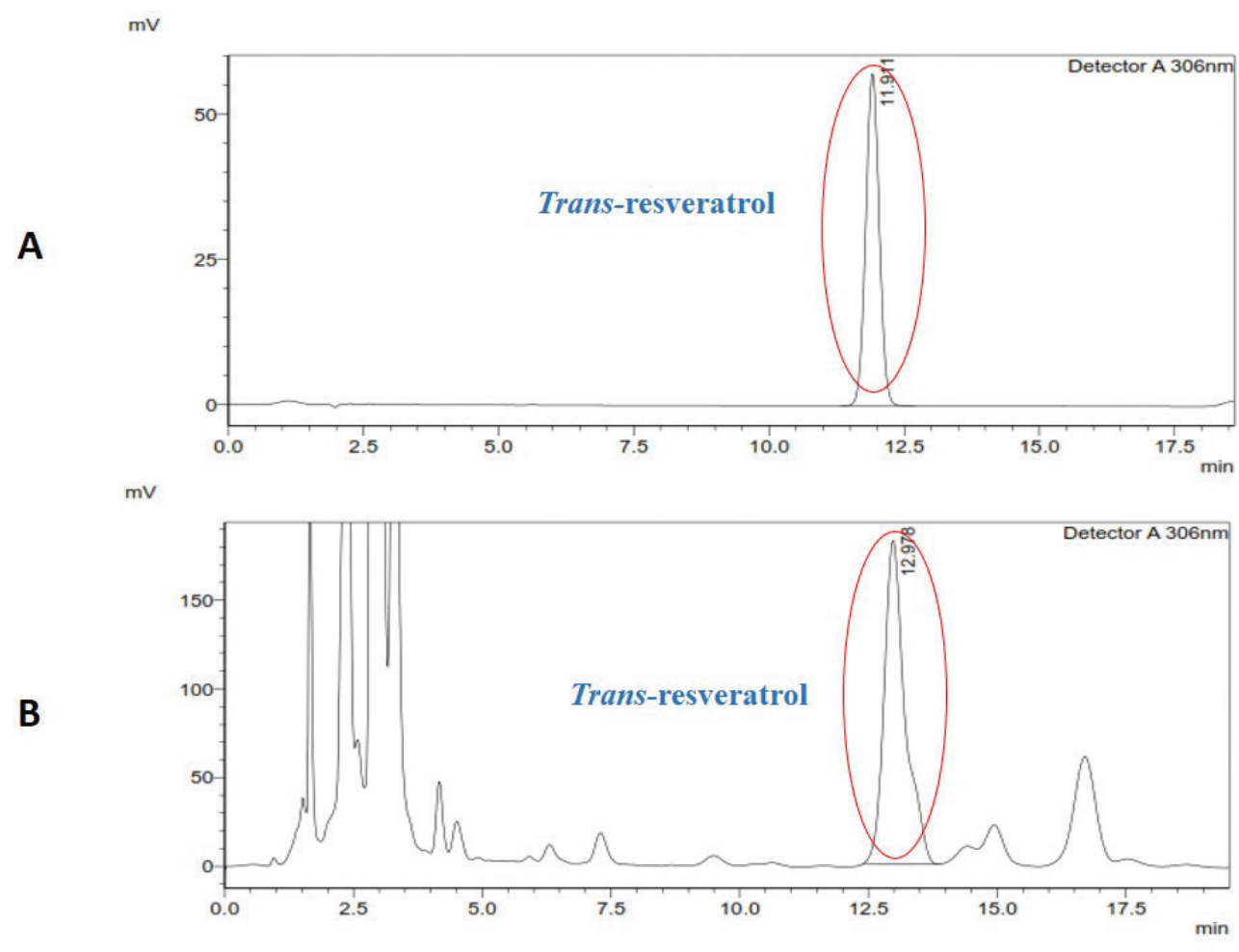

Figure 8. The Results Chromatogram of Trans-Resveratrol with HPLC Method

resveratrol in the water and the presence of a large amount of surfactant. Good solubility of trans-resveratrol in the lipid matrix, which has the good emulsifying capacity, was another factor that contributes to the high entrapment efficiency of SLN system. This study proved that SLN provides excellent occlusive effects, which can help reduce skin moisture loss and increase water content in the stratum corneum, causing impaired skin barrier function and increased diffusion coefficient (Sun et al., 2014).

\section{CONCLUSION}

IL-MSE loaded to SLNs system can be prepared successfully by using modified high-pressure homogenization as proved in previous studies. The surface structure of the formulation was spherical and rough. The system could provide good entrapment efficiencies over $89 \%$, and the mean size was in the range of 794 $\mathrm{nm}$. The in vitro studies about the data of skin permeation showed that IL-MSE loaded SLN-based gels provide sustained release of trans-resveratrol. According to this study showed that the SLN system has good penetration through the skin. This research can prove that the SLNs can be further developed as a topical delivery carrier in increasing the penetration of trans-resveratrol to the skin.

\section{ACKNOWLEDGMENT}

This research was supported by the Ministry of Research and Technology, The Republic of Indonesia and the Directorate of Research and Community Service (DRPM), Universitas Indonesia through PITTA 2017.

\section{CONFLICT OF INTEREST}

The authors declare that they have no conflicts of interest.

\section{ABBREVIATION:}

IL-MSE ionic liquid-melinjo seed extract;

MAE microwave-assisted extraction;

RSM response Surface Methodology;

HPLC high-performance liquid chromatography;

SEM scanning electron microscopy;

SLNs solid lipid nanoparticles;

PS particle size;

PDI polydispersity index;

EE entrapment efficiency;

HPH high-pressure homogenization.

\section{REFERENCES}

Ansari KA, Vavia PR, Trotta F, Cavalli R. Cyclodextrin-based nanosponges for delivery of resveratrol: in vitro characterisation, stability, cytotoxicity and permeation study. AAPS PharmTech, 2011; 12(1):279-86.

Ayuningtyas, IN, Rahmawati, M, Sutriyo, Mun'im, A. Optimization of ionic liquid-based microwave assisted extraction to obtain trans-resveratrol from Gnetum gnemon L. seeds. J Young Pharm, 2017; 9(4):457-62.

Barua CC, Haloi P, Barua IC. Gnetum gnemon Linn. : a comprehensive review on its biological, pharmacological and pharmacognostical potentials. Int J Pharmacogn Phytochem Res, 2015; 7(3):531-9.

Casazza AA, Aliakbarian B, Mantegna S, Cravotto G, Perego P. Extraction of phenolics from Vitis vinifera wastes using non-conventional techniques. J Food Eng, 2010; 100(1):50-5.

Davidov-Pardo G, McClements DJ. Resveratrol encapsulation: designing delivery systems to overcome solubility, stability and bioavailability issues. Trends in Food Sci Technol, 2014; 38(2):88-103.

Detoni CB, Souto GD, Luiza A, Pohlmann AR, Guterres SS. Photostability and skin penetration of different E-resveratrol-loaded supramolecular structures. Photochem Photobiol, 2012; 88(4):913-21. 
Gokce EH, Korkmaz E, Dellera E, Sandri G, Cristina Bonferoni M, Ozer O. Resveratrol-loaded solid lipid nanoparticles versus nanostructured lipid carriers: evaluation of antioxidant potential for dermal applications. Int J Nanomed, 2012; 7:1841-50.

Kato E, Tokunaga Y, Sakan F. Stilbenoids isolated from the seeds of melinjo (Gnetum gnemon L.) and their biological activity. J Agric Food Chem, 2009; 57(6):2544-9.

Lee TH, Seo JO, Baek S, Kim SY. Inhibitory effects of resveratrol on melanin synthesis in ultraviolet B-induced pigmentation in Guinea Pig Skin. Biomol Ther, 2014; 22(1):35-40.

Liazid A, Palma M, Brigui J, Barroso CG. Investigation on phenolic compounds stability during microwave-assisted extraction. J Chromatogr A, 2007; 1140(1-2):29-34.

Mehnert W, Mader K. Solid lipid nanoparticles: production, characterization and applications. Adv Drug Deliv Rev, 2001; 47:165-96.

Muller, RH. Zetapotential and Partikelladung in der Laborpraxis. 1st edition, Wissenschaftliche Verlagsgesellschaft, Stuttgart, Germany, 1996.

Muller RH, Radtke M, Wissing SA. Solid lipid nanoparticles (SLN) and nanostructured lipid carriers (NLC) in cosmetic and dermatological preparations. Adv Drug Deliv Rev, 2002; 54:131-55.

Rigon RB, Fachinetti N, Severino P, Santana MHA. Skin delivery and in vitro biological evaluation of trans-resveratrol-loaded solid lipid nanoparticles. Molecules, 2016; 21:116-219.

Sarathchandiran I. A review on nanotechnology in solid lipid nanoparticles. Int J Pharm Dev Technol, 2012; 2(1):45-61.

Scognamiglio I, Stefano D De, Campani V, Mayol L, Carnuccio R, Fabbrocini G, Ayala F, La Rotonda MI, De Rosa G. Nanocarriers for topical administration of resveratrol : a comparative study. Int J Pharm, 2013; 440(2):179-87.

Sun R, Zhao G, Ni S, Xia Q. Lipid-based nanocarriers with different lipid compositions for topical delivery of resveratrol: comparative analysis of characteristics and performance. J Drug Deliv Sci Technol, 2014; 24(6):591-600.
Stetefeld J, McKenna SA, Patel TR. Dynamic light scattering: a practical guide and applications in biomedical sciences. Biophys Rev, 2016; 8:409-27.

Tani H, Hikami S, Iizuna S, Yoshimatsu M, Asama T, Ota $\mathrm{H}$, Kimura Y, Tatefuji T, Hashimoto K, Higaki K. Pharmacokinetics and safety of resveratrol derivatives in humans after oral administration of melinjo (Gnetum gnemon L.) seed extract powder. J Agric Food Chem, 2014; 62(8):1999-2007.

Teja SPS, Damodharan N. $2^{3}$ full factorial model for particle size optimization of methotrexate loaded chitosan nanocarriers: a design of experiments (DoE) approach. BioMed Res Int, 2018; 2018:1-9.

Trinovita, E, Sigalingging, E, Saputri, FC, Mun'im, A. Optimization of ionic liquid 1-Butyl-3-Methylimidazolium Hexafluorophosphate ([Bmim] $\left.\mathrm{PF}_{6}\right)$-based microwave assisted extraction method for gamma oryzanol from rice bran (Oryza sativa L.). J Appl Pharm Sci, 2017; 7(12):008-13.

How to cite this article:

Trinovita E, Rachmawati M, Sutriyo S, Mun'im A. In vitro penetration activity of ionic liquid-Gnetum gnemon seed extract loaded solid lipid nanoparticles. J Appl Pharm Sci, 2019; 9(10):009-016 